\title{
Towards a Protocol for the Attachment of Semantic Descriptions to Grid Services
}

\author{
Simon Miles, Juri Papay, Terry Payne, Keith Decker and Luc Moreau \\ School of Electronics and Computer Science \\ University of Southampton \\ Southampton, SO17 1BJ, UK \\ \{sm, jp, trp, ksd, L.Moreau\}@ecs.soton.ac.uk
}

\begin{abstract}
Service discovery in large scale, open distributed systems is difficult because of the need to filter out services suitable to the task at hand from a potentially huge pool of possibilities. Semantic descriptions have been advocated as the key to expressive service discovery, but the most commonly used service descriptions and registry protocols do not support such descriptions in a general manner. In this paper, we present a protocol, its implementation and an API for registering semantic service descriptions and other task/user-specific metadata, and for discovering services according to these. Our approach is based on a mechanism for attaching structured and unstructured metadata, which we show to be applicable to multiple registry technologies. The result is an extremely flexible service registry that can be the basis of a sophisticated semantically-enhanced service discovery engine, an essential component of a Semantic Grid.
\end{abstract}

\section{Introduction}

Service discovery is a difficult task in large scale, open distributed systems such as the Grid and Web, due to the potentially large number of services advertised. In order to characterise their needs, users typically specify the complex requirements they have of a service, including demands on its functionality, quality of service, security, reputation and so on. These requirements cannot be fully expressed in the constrained information models provided by existing service directory technologies such as UDDI (the de facto standard for Web Services registry), the OGSA Registry [7] or the Jini lookup service [1].

More demandingly, user requirements may contain information that assumes an understanding of particular application domains and that is meant to effect the outcome of the discovery process. For example, a bioinformatician may search for services that process "expressed sequence tags" but also that operate on "nucleotide sequences". The knowledge that the latter biological concept is a more general form of the former is known by the user, but is unlikely to be expressible in either the query or the standard descriptions of useful services.

On the other hand, there may be information that could usefully be utilised in the discovery process, but is not or cannot be published by the service provider. For example, recommendations about which services to use may come from one member of an organisation, and could be used by their collaborators.

Finally, there are functional entities other than services that need to be publicised. For example, workflow scripts describe how to compose services together to get a useful composite behaviour. They are location-independent descriptions of service use and should be publishable in the same registries as services since they provide comparable function, i.e. they are invocable, take inputs and return outputs (this equivalence is recognised explicitly in DAML-S [6]). 
Given the plethora of services and sophistication of user requirements, many have advocated the use of semantic descriptions that qualify functional and nonfunctional characteristics of services in a manner that is amenable to automatic processing $[3,6,14]$. Broadly, such semantic descriptions offer a powerful and easyto-use form of extensibility, which lacks in existing Grid service discovery technologies. Against this background, we have designed a system that allows the attachment of semantic information so as to provide a solution to the shortcomings of existing service directory technologies. In this paper, we report on our experience of designing such a system, specifically focusing on the following contributions.

- We have developed a protocol for attaching metadata to registered service descriptions, and for querying over service descriptions and their metadata.

- We have implemented a UDDI-compatible service registry that allows metadata to be attached to various parts of the service description, such as the services themselves, their operations, their inputs and their outputs (as specified in WSDL documents describing service interfaces).

- An extensible programmatic interface (API) provides clients with an easy way to access this information, whether it is held in a remote Web Service or locally.

- We demonstrate with a common information model that our attachment methodology applies to multiple discovery technologies in a single directory, including UDDI, DAML-S and BioMOBY.

These developments are part of the architecture developed by the myGrid project (www.mygrid.org.uk). They constitute a core component, which we use in practice to publish semantic service descriptions and reason over them. The reader interested in issues related to semantic reasoning over the service descriptions stored in our registry in myGrid is referred to [9], and in performance appraisals of our service to $[10]$.

In this paper, we will first examine the limitations of existing approaches (Section 2 ), then look at how we overcome these by allowing for service descriptions by a metadata attachment mechanism (Section 3). We then examine the protocol by which a client can register and query over metadata (Section 4), and in Section 5 we discuss how our services can help in overcoming some of the problems mentioned above. Finally, we discuss the implications of our work and its current and future developments in Section 6.

\section{Limitation of Existing Approaches}

The UDDI service directory (Universal Description, Discovery, and Integration) [12] has become the de-facto standard for service discovery in the Web Services community. Service queries are typically white or yellow pages based: services are located using a description of their provider or a specific classification (taken from a published taxonomy) of the desired service type. Service descriptions in UDDI are composed from a limited set of high-level data constructs (Business Entity, Business Service etc.) which can include other constructs following a rigid schema. Some of these constructs, such as tModels, Category Bags and Identifier Bags, can be seen as metadata associated with the service description. However, while useful in a limited way, they are all very restrictive in scope of description and their use in searching the directory. In particular, services are not the only entities that need to be classified. For instance, classifications can also be defined for individual operations or their argument types. However, it is not convenient to use searching mechanisms for services that are distinct from those for their argument types. Likewise, a tModel's reference to an external technical specification, such as a WSDL file describing a service interface, also implies that a different mechanism is required for reasoning over 
service interfaces. These are clear restrictions of the facilities offered for attaching metadata to entities in UDDI service descriptions.

Related to this, some uses of WSDL, the interface definition language of Web Services, suffer from limitations, as illustrated by Figure 1 displaying the interface of an existing bioinformatics service called BLAST. BLAST is a widely used analysis tool that establish the similarity of a DNA sequence of unknown function to already annotated ones to help the biologist gain an understanding of its possible function. We use BLAST in a data-intensive bioinformatics Grid application, which aims to make an educated guess of the gene involved in a disease and to design an experiment to be realised in the laboratory in order to validate the guess. The BLAST service is invoked, as part of a workflow enacted over the Grid, on a large number of sequences without user intervention.

The interface of Figure 1 identifies a portType composed of one operation, which takes an input message comprising two message parts in 0 and in1. These parts are required to be of type string, but the specification does not tell us what the meaning of these strings is supposed to be. These are supposed to be sequences, for which many formats are supported. This example was chosen because it precisely illustrates limitations of some existing service descriptions. While this interface specification could easily be refined by using an XSD complex type [4], it is unrealistic to assume that all services in an open Grid environment will always be described with the appropriate level of detail. Moreover, should it be so, we cannot expect all service providers to always use type definitions expressed with the terms of reference adopted by a given user.

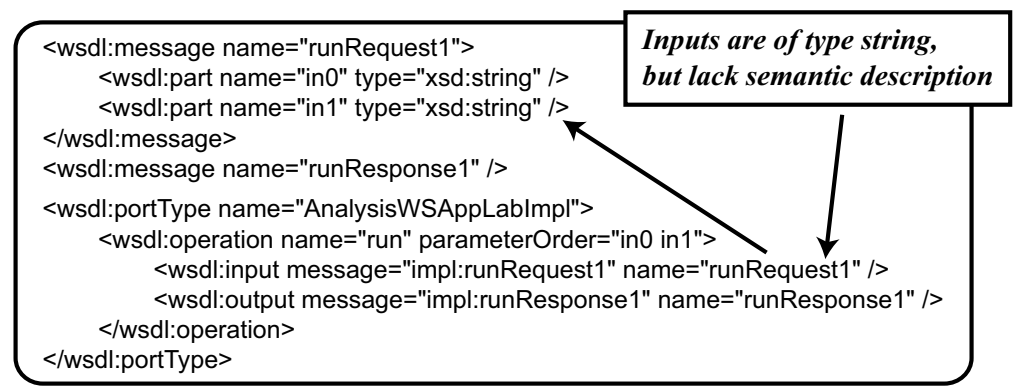

Fig. 1. Basic Local Alignment Search Tool (BLAST) Interface Excerpt

Other problems related to the rigid nature of UDDI and WSDL, and the lack of metadata attachment capabilities, can be seen when looking at the uses to which they are put. A UDDI query typically returns a list of available services, from which a subset may conform to a known and/or informally agreed upon policy and thus can be invoked. Such approaches work well within small, closed communities, where a priori definitions of signatures and data formats can be defined. However, across open systems such as the Grid, no assumption can be made about how desired services are described, how to interact with them, and how to interpret their corresponding results. Additionally, service providers typically adopt different ways to model and present services, often because of the subtle differences in the service itself. This raises the problem of semantic inter-operability, which is the capability of computer systems to meaningfully operate in conjunction with one another, even though the languages or structures with which they are described may be different. Semantic discovery is the process of discovering services capable of semantic inter-operability. 
For example, the BLAST service, discussed earlier, is made available to clients in very different ways. A deployment of the service by IBM can perform in four steps the same function that a service deployed at the European Bioinformatics Institute performs in three steps, due to the differences in setting up sessions and in providing each necessary piece of data. However, overall, they both take the same information and perform the same task and so can both semantically inter-operate with a service providing DNA sequences.

Also, UDDI provides no data structures to represent either the abstract or concrete details contained within a WSDL document, but only a standard way to express that a service implements a particular wSDL interface. A new proposal allows tModels to reference specific bindings and port types [5]. However, this extension still does not provide access to, or queries over, operations or messages, which would allow the discovery of services capable of specific operations.

DAML-S attempts a full description of a service from the point of view that it is some process that can be enacted to achieve a goal. A full DAML-S service description incorporates three component perspectives: an abstract description of the service from the AI planning view, which includes inputs, outputs, preconditions, and effects of a service (the service profile); the workflow view of the more primitive services needed to accomplish a complex goal (the service process); the mapping of the atomic parts of this workflow to their concrete WSDL descriptions (the service grounding). Of interest to this paper is DAML-S' capability to attach semantic annotations to service descriptions. First, by its ontological nature, the DAML-S ontology may be subclassed to provide new information about services such as, e.g. the task performed by a service or the algorithm it relies upon, as discussed in [14]. DAML-S provides an alternate mechanism that allows service publishers to attach semantic information to the parameters of a service. Indeed, the argument types referred to by the profile input and output parameters are semantic. Such semantic types are mapped to the syntactic type specified in the WSDL interface by the intermediary of the service grounding. We feel that such a mechanism is a step in the right direction, but it is convoluted (in particular, because the mapping from semantic to syntactic types involves the process model, which we did not discuss). It also has some limitations since it only supports semantic annotations provided by the publisher, and not by third-party annotators; furthermore, a profile only supports one semantic description per parameter and does not allow multiple interpretations. Finally, such semantic annotations are restricted to input and output parameters, but may not be applied, in a similar manner, to other elements of a WSDL interface specification.

BiomOBY [13] is a service discovery architecture based on a view of a service as an atomic process or operation that takes a set of inputs and produces a set of outputs. The service, inputs and outputs can all be characterised by semantic types; inputs and outputs will also have syntactic types. So, for example, a service provider may register a BLAST service to take (semantically) nucleotide sequences (syntactically, simple strings) and perhaps (semantically) a BLAST e-value cutoff (syntactically, a real number), and produce a set of matching sequences and e-values. It is limited in that it does not support the UDDI protocol, so specialist clients have to be developed, and it cannot have general extension of service descriptions, so, for example, the attachment of quality of service ratings or structured descriptions to service adverts is not supported.

\section{Extending Service Descriptions}

Having discussed the limitations of existing technologies, we now focus on the capabilities of our service directory. This directory allows for extension of service descriptions by adding metadata attachments. A metadata attachment is an extra 
piece of data giving information about an existing entity in a service description, and is explicitly associated with that entity. Entities to which metadata can be attached include the service itself, an operation supported by the service, an input or output type of an operation invocable on the service. The metadata is attached by calls to the service directory after publishing, with reference to the entity to which the metadata should be attached. To establish that this attachment mechanism is generic, we have applied it to the service descriptions supported by UDDI, WSDL, DAML-S and BioMOBY, which we all encode with a common information model.

We have adopted RDF triples [11] to represent all descriptions of services. RDF (Resource Description Framework) is an XML data format for describing Web and Grid resources and the relations between them. Triples are simple expressions of the relations between resources, consisting of a subject, a relation (or property) and an object. All our triples are stored in a triple store, which is a database whose interface and implementation are specially designed to hold such triples. Specifically, we rely on the Jena implementation [8] of such a triple store.

To illustrate our general mechanism, we consider different kinds of metadata attachment, for which we found practical uses in our Grid application: (i) attaching ratings to services; (ii) attaching functionality profiles to services; (iii) attaching semantic types to operation arguments. Ratings can provide users with assessments from experts on the value of a particular service; functionality profiles can be used to refine a search to exactly those services that are relevant; and semantic types allow clients to ask whether services are applicable to the type of data they have (so overcoming the limitations of WSDL described above). Our presentation is based on examples that were generated by dumping the contents of our service directory; it relies on the N3 format [2], which we have chosen for its readability. For example, in Figure 2, we show the representation of a service annotated by two numerical ratings, with different values, and provided by different authors at different times. The service is described by many pieces of information from the standard UDDI model such as its service key (the value to the right of uddi:hasServiceKey), and by two pieces of metadata attached to it (under uddi:hasMetadata). Each piece of metadata has a type (in this case, both are of type mygrid:NumericRating), a value (the rating itself) and two pieces of provenance information. The provenance information is the time and date at which the metadata was published and the name of the publisher. Such information is particularly useful in a registry allowing third parties to attach ratings because it can be used to identify the origin of an annotation.

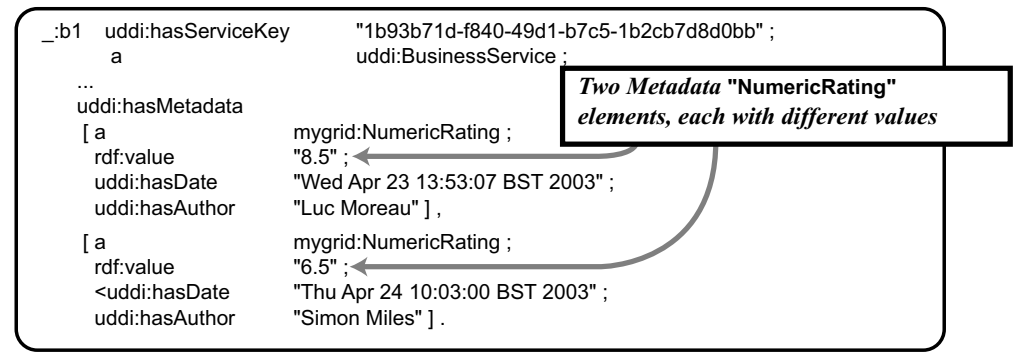

Fig. 2. Rating Attachment (N3 Notation)

In myGrid, we describe services by a service profile [14] specifying which particular type of process a service uses to perform its task (uses_method), which application domain specific task they perform (perform_task), which resources they use (uses_resources) and what application they are wrapping (is_function_of). 
A relevant excerpt of the service directory contents is displayed in Figure 3, with b1 denoting a service and Pe577955b-d271-4a5b-8099-001abc1da633 the "myGrid profile". This is useful because it presents clients with information that is concise but matches their knowledge of the service's application domain (bioinformatics in this case), and can be reasoned about by other semantic technologies using expert knowledge on the application domain available on the Grid.

In this specific example, it should be noted that the objects of the different triples, nucleotide_sequence_database, mygrid_bioinformatics_ primitive_service_operation, pairwise_local_aligning, and blastn, are all concepts of a published ontology of the bioinformatics domain [14], which is not directly held in the repository, but is used for semantic reasoning over that domain. Semantic reasoning may allow a discovery mechanism to, for example, return a service annotated with blastn in response to an alignment request, since the latter subsumes the former [9].

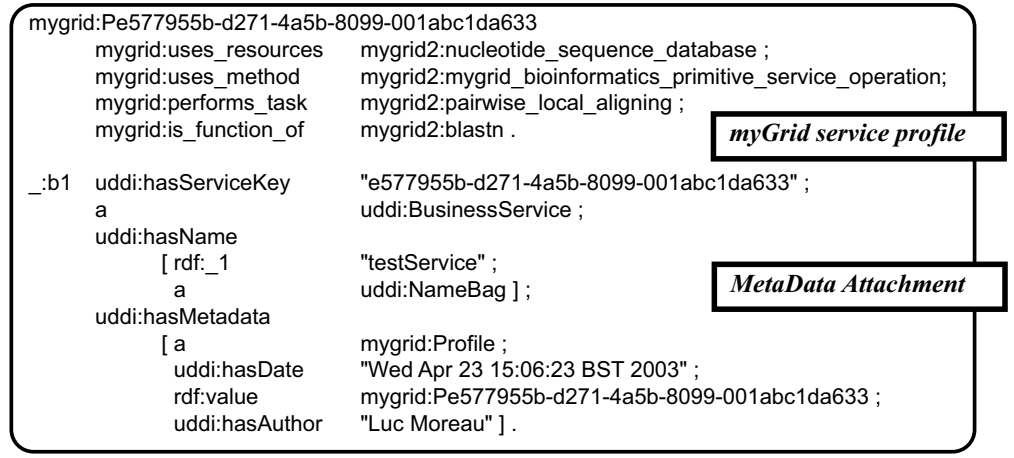

Fig. 3. Attachment of a myGrid profile (N3 Notation)

In scientific application areas, the scientists often want to discover services that analyse the data they have obtained from experiments. We showed that the description of input and output syntax, given for example by XSD definitions in WSDL, is not always adequate to determine whether the service is applicable for consuming or producing data of a particular semantic type. Figure 4 illustrates a semantic description of parameter in 0 declared in the interface of Figure 1 . The node $r d f: \_1$ denotes the message part with name in 0 . It is given a metadata attachment, with value mygrid2:nucleotide_sequence_data, which again refers to a term in the ontology of bioinformatics concepts [14].

\section{Service Directory Protocol and Implementation}

Protocol The protocol to publish metadata and discover services according to metadata was designed in a similar style to the UDDI protocol, so that UDDI clients could easily be extended to support such features. It is not possible to present the complete protocol in this paper. Instead, we refer the reader to the full set of commented interfaces, which can be accessed from www.ecs.soton.ac.uk/ ${ }^{\sim}$ sm/myGrid/Views/. As an illustration, Figure 5 shows some of the methods that allow the attachment of metadata, respectively to a business service, to a business entity, to an operation and to a message part. All these methods not only attach some metadata to the respective entity, but also add the aforementioned provenance information such as author and date of creation. The associated metadata can be structured or 


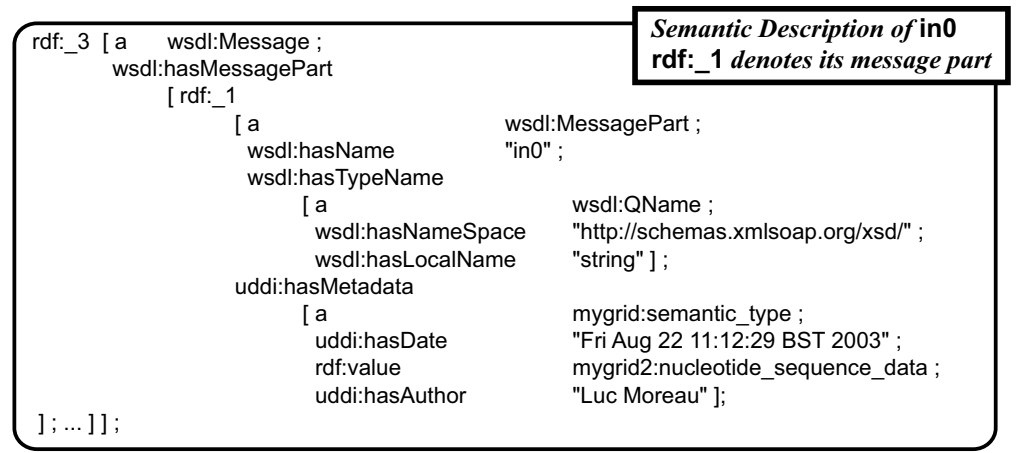

Fig. 4. Attachment of Semantic Types to Arguments (N3 Notation)

unstructured. Symmetrically, services can be discovered by using a metadata filtering mechanism. An example of metadata-based search method appears in Figure 5. Given some metadata, the findServiceByMetadata function returns the list of services that are annotated with such a metadata.

We support semantic discovery by allowing the metadata structure to contain terms of an ontology, which triggers ontological-based reasoning, the description of which is beyond the scope of this paper, but more details can be found in [9].

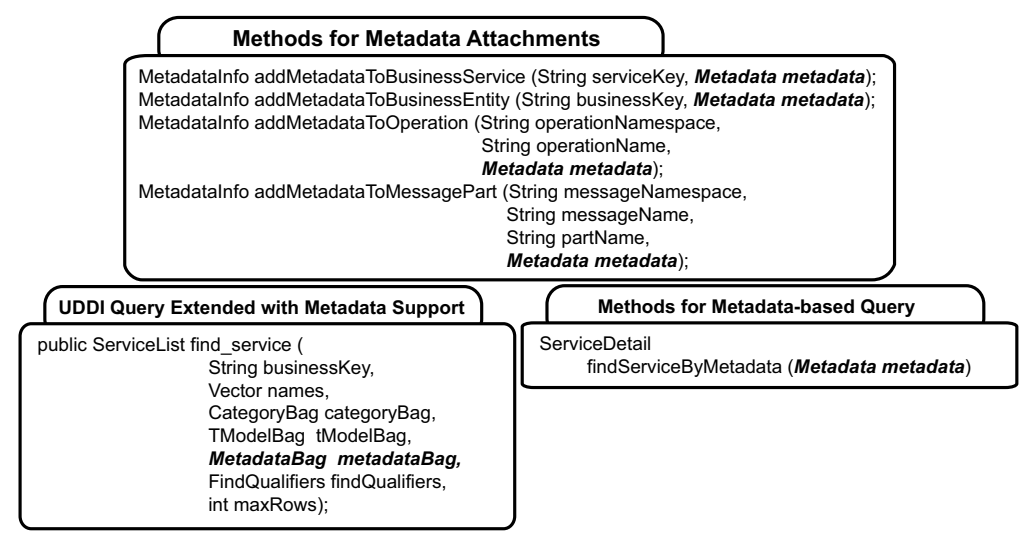

Fig. 5. Metadata Attachment Methods

The benefit of our approach is the ability to extend some existing interfaces in an incremental manner, so as to facilitate an easier transition to semantic discovery for existing clients. For instance, we have extended the UDDI find_service method to support queries over metadata that would have been attached to published services. In the method specification of Figure 5, metadataBag, a new criterion for filtering services is introduced, which contains a set of metadata that a service must satisfy.

API We also provide a client-side library, or API, that makes it easy to use the protocol to communicate with the registry. Figure 6 illustrates how our API can be used to attach a semantic description to the first input of the runRequest1 operation of Figure 1. The arguments of the addMetadataToMessagePart are the namespace of the service interface (an excerpt of which appears in Figure 1), the operation name (runRequest1), the parameter name (in0), and an object of 
type Metadata, whose type and values have been initialised to semantic_type and nucleotide_sequence_data respectively.

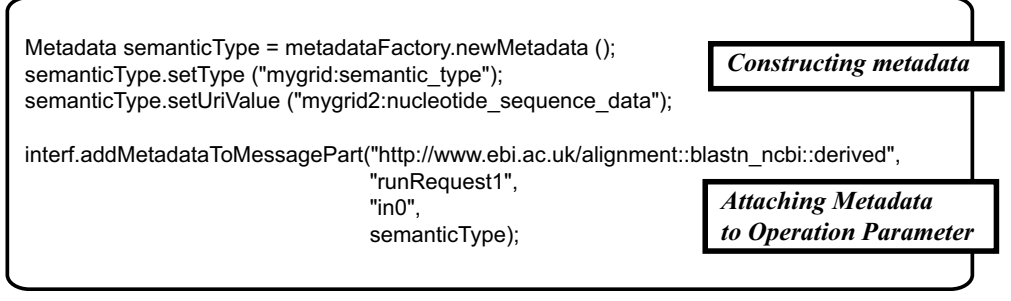

Fig. 6. Registering a parameter semantic type

As all the information is represented in a triple store, a more direct interface to the triple store allows users to query the service directory using the RDQL query language [8]. An API that allows users to store triples in the triple store is also provided.

Several interfaces currently provide access to our general information model. Some of them preserve compatibility with the existing UDDI standard, and ensure inter-operability within the Web Services community. Others, such as the interface to the triple store, directly expose the information model, and offer a powerful and radically different way of discovering services through the RDQL interface. While such functionality is very useful, its radically different nature does not offer a smooth transition for clients implementors wishing to adopt semantic discovery.

Implementation We have implemented the protocol in a registry, within which all service descriptions and metadata attachment are expressed as RDF triples and stored in the Jena triple store. We have designed and implemented a set of interfaces to the Jena triple store in order to offer a service directory functionality. Figure 7 depicts excerpts of class and collaboration diagrams. We see that a service directory implements a series of factory methods to create instances of interfaces to the triple store. The triple store is passed as argument to the factory methods and is itself created by a store factory; different implementations of a store may exist, in memory or in a relational database [8].

There are four service directory interfaces shown: PublishUDDI and InquiryUDDI allow publishing and discovery of services using the UDDI protocol; InquiryMetadata provides searching based on the metadata attached to service description entities; and WSDL contains methods for publishing WSDL files to which metadata can be attached.

\section{Discussion}

We can now return to the initial Grid service discovery requirements presented in the introduction, and show how the work presented above addresses each.

Automated Service Discovery Using our service directory, arbitrary metadata can be attached to service descriptions and then programmatically discovered. Metadata can be simple strings and URIs referring to other resources, such as ontological concepts. It can also be arbitrarily structured by giving typed relations between pieces of metadata using triples. This can be attached to service descriptions using the methods and technologies discussed in Section 4. 

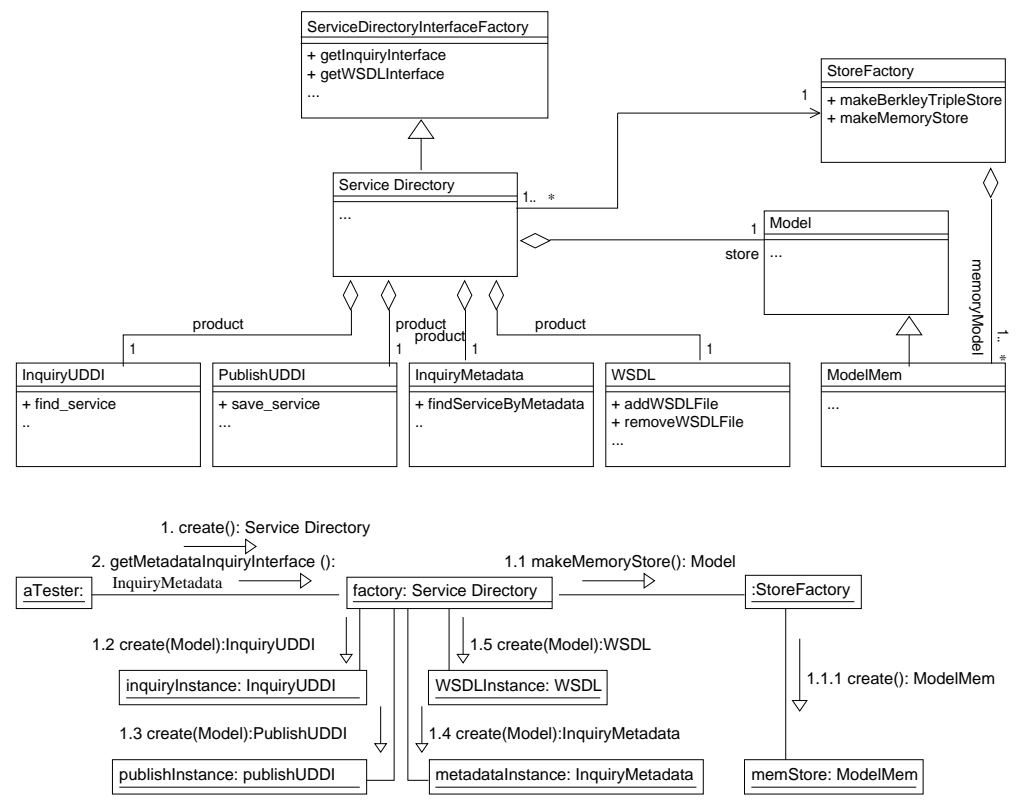

Fig. 7. Class and Collaboration Diagram

Expert Knowledge Queries Users can present queries that refer to terms in the domain in which they have expertise. Using the semantic terms that can be attached to service descriptions using our technology along with the expert knowledge encoded in relevant ontologies and semantic reasoning, discovery of services will be widened or constrained based on the experience already built up by other knowledgeable professionals in the field.

Recommendations and Personal Opinions Recommendations regarding services, and opinions on their suitability and quality from users can be attached as metadata to service descriptions and used in discovery. Clearly, this information may not be appropriate for storing in the public registries in which services are published. We are currently working on a factory to create "views" over existing service registries.

Publishing of Other Process Descriptions Workflow scripts and other locationindependent processes can be published and discovered in our directory. Because they can be discovered and executed using the appropriate tools, they are directly comparable to services invocation. In terms of interfaces, workflows and parameterised queries both take inputs and provide outputs, each of which may be semantically annotated to enhance discovery.

\section{Conclusion}

In this paper, we have presented a protocol to publish semantic descriptions about services in order to promote semantic inter-operability. Our approach relies on a metadata attachment mechanism, capable of attaching metadata to any entity within a service description. Such metadata need not be published by service providers but can be published by third-party users. Our design extends the standard interface UDDI to provide semantic capabilities, hereby offering a smooth transition to semantic discovery for UDDI clients. We have used these facilities to register service descriptions as specified by the myGrid ontology [14]. Our future work will focus on providing service descriptions to Grid Services. This has extra 
demands on top of those for Web Services, due to Grid Services being created by factories, having lifetimes and server-side metadata (service data elements). Grid Service factories, in particular, require extra information to be stored in a service registry to allow clients to identify them as such and be informed as to how to use them to create the Grid Services they need.

\section{Acknowledgement}

This research is funded in part by EPSRC myGrid project (reference GR/R67743/01). Keith Decker from the University of Delaware was on sabbatical stay at the University of Southampton when this work was carried out. We acknowledge Carole Goble, Phillip Lord and Chris Wroe for their contributions to discussion on the work presented in this paper.

\section{References}

1. Arnod, O'Sullivand, Scheifler, Waldo, and Wollrath. The Jini Specification. Sun Microsystems, 1999.

2. Tim Berners-Lee. Notation 3. http://www.w3.org/DesignIssues/Notation3, 1998.

3. Tim Berners-Lee, James Hendler, and Ora Lassila. The Semantic Web. Scientific American, 284(5):34-43, 2001.

4. Paul V. Biron and Ashok Malhotra. Xml schema part 2: Datatypes. http://www.w3.org/TR/xmlschema-2/, May 2001.

5. John Colgrave and Karsten Januszewski. Using wsdl in a uddi registry (version 2.0). http://www.oasis-open.org/committees/uddi-spec/doc/draft/uddi-spec-tctn-wsdl-20030319-wd.htm, 2003.

6. DAML-S Coalition:, A. Ankolekar, M. Burstein, J. Hobbs, O. Lassila, D. McDermott, D. Martin, S. McIlraith, S. Narayanan, M. Paolucci, T. Payne, and K. Sycara. DAMLS: Web Service Description for the Semantic Web. In First International Semantic Web Conference (ISWC) Proceedings, pages 348-363, 2002.

7. Ian Foster, Carl Kesselman, Jeffrey M. Nick, and Steven Tuecke. The Physiology of the Grid - An Open Grid Services Architecture for Distributed Systems Integration. Technical report, Argonne National Laboratory, 2002.

8. Jena semantic web toolkit. http://www.hpl.hp.com/semweb/jena.htm.

9. Phillip Lord, Chris Wroe, Robert Stevens, Carole Goble, Simon Miles, Luc Moreau, Keith Decker, Terry Payne, and Juri Papay. Semantic and Personalised Service Discovery. In W. K. Cheung and Y. Ye, editors, WI/IAT 2003 Workshop on Knowledge Grid and Grid Intelligence, pages 100-107, Halifax, Canada, October 2003. Department of Mathematics and Computing Science, Saint Marys University, Halifax, Nova Scotia, Canada.

10. Simon Miles, Juri Papay, Vijay Dialani, Michael Luck, Keith Decker, Terry Payne, and Luc Moreau. Personalised grid service discovery. IEE Proceedings Software: Special Issue on Performance Engineering, 150(4):252-256, August 2003.

11. Resource Description Framework (RDF). http://www.w3.org/RDF/, 2001.

12. Universal Description, Discovery and Integration of Business of the Web. www .uddi .org, 2001.

13. MD Wilkinson and M. Links. Biomoby: an open-source biological web services proposal. Briefings In Bioinformatics, 4(3), 2002.

14. Chris Wroe, Robert Stevens, Carole Goble, Angus Roberts, and Mark Greenwood. A suite of daml+oil ontologies to describe bioinformatics web services and data. International Journal of Cooperative Information Systems, 2003. 